\title{
SUCCESSIVE DERIVATIVES OF ENTIRE FUNCTIONS
}

\author{
SIMON HELLERSTEIN ${ }^{1}$ AND JACK WILLIAMSON ${ }^{2}$
}

\begin{abstract}
We show that if $f$ is a real entire function which has, along with each of its derivatives, only real nonpositive zeros, then either $f(z)=c e^{o z}, c$ and $\sigma$ real constants, or
\end{abstract}

$$
f(z)=c z^{m} e^{\sigma z} \prod_{n}\left(1+\frac{z}{\left|z_{n}\right|}\right)
$$

where $\sigma>0$ and $\Sigma_{n}\left|z_{n}\right|^{-1}<\infty$.

This note concerns a subclass of the class $\mathscr{U}_{0}$ of entire functions $g$ of the form

$$
g(z)=c z^{m} e^{-\gamma z^{2}+\sigma z} \prod_{n}\left(1-\frac{z}{z_{n}}\right) e^{z / z_{n}}
$$

where $c$ is a constant, $\gamma \geqslant 0, \sigma$ and the $z_{n}$ are real, and $\Sigma_{n}\left|z_{n}\right|^{-2}<\infty$. The subclass of $\mathscr{U}_{0}$ that we will be interested in is the class $\mathscr{U}$ of entire functions $f$ of the form

$$
f(z)=c z^{m} e^{\sigma z} \prod_{n}\left(1+\frac{z}{\left|z_{n}\right|}\right)
$$

where $\sigma \geqslant 0$ and $\Sigma_{n}\left|z_{n}\right|^{-1}<\infty$.

The class $\mathscr{U}_{0}$ (often called the Laguerre-Pólya class) and its subclass $\mathscr{U}$ are of special interest since classical theorems of Laguerre [3] and Pólya [4] assert that $f \in \mathcal{U}_{0}(\mathscr{U})$ if and only if $f$ can be uniformly approximated on discs about the origin by a sequence of polynomials with only real (real nonpositive) zeros. A corollary of their results is: $f \in \mathscr{U}_{0}(U)$ implies $f^{(n)} \in \mathscr{U}_{0}(U)$, $n=1,2, \ldots ;$ in particular, $f \in \mathscr{U}_{0}(\mathscr{Q})$ implies $f^{(n)}$ has only real (real nonpositive) zeros $n=1,2, \ldots$ In 1915, Pólya [6] (see also [5]) asked whether the following converse assertion holds: If a (constant multiple of a) real entire function $f$ (i.e., $z$ real implies $f(z)$ real) and each of its derivatives $f^{(n)}, n=1,2, \ldots$ have only real (real nonpositive) zeros is $f \in \mathcal{Q}_{0}(\mathcal{Q})$ ?

Pólya showed [5], [6] that if $f(z)=P(z) e^{Q(z)}$ where $P$ and $Q$ are polynomials, then the answer to this question is affirmative. Recently the authors

Received by the editors December 13, 1976 and, in revised form, March 21, 1977.

AMS (MOS) subject classifications (1970). Primary 30A66.

Key words and phrases. Real entire function, derivative, genus of a canonical product.

${ }^{1}$ Supported in part by NSF grant MPS 75-07000.

${ }^{2}$ Supported in part by NSF grant MPS 75-07098. 
established [1], [2] the full conjecture for $\mathscr{Q}_{0}$ with the following

THEOREM A. Let $f$ be a (constant multiple of a) real entire function. If $f, f^{\prime}$, and $f^{\prime \prime}$ have only real zeros, then $f \in Q_{0}$.

In this note we use this result to establish the conjecture for $\mathscr{U}$ with the

THEOREM B. Let $f$ be a (constant multiple of a) real entire function. If $f$ and $f^{(n)}$ have only real nonpositive zeros $n=1,2, \ldots$, then either $f(z)=c e^{\sigma z}$, o real, or $f \in \mathcal{Q}$.

Before proving Theorem B, we remark that its hypotheses cannot be weakened by requiring only that $f$ and $f^{(n)}, n=1,2, \ldots, N$, for some $N$ have only real nonpositive zeros - see Remark 2 below.

Proof of Theorem B. From Theorem A we see that

$$
f(z)=c z^{m} e^{-\gamma z^{2}+o z} \prod(z)
$$

where $c$ is a constant which, without loss of generality, we take to be 1 , and where $\Pi(z)$ is a canonical product of genus $\leqslant 1$ with only negative zeros. Thus, it only remains to show that if $f(z) \neq c e^{o z}$, then (i) $\gamma=0$, (ii) $\Pi$ is of genus 0 , and (iii) $\sigma \geqslant 0$. To do this we first observe that there is no loss in generality in assuming that $f$ and $f^{(k)}, k=1,2, \ldots$, have only negative zeros. Indeed, if $f^{(k)}$ has a zero at the origin for some $k\left(f^{(0)}=f\right)$, consider $g(z)=f(z+\varepsilon)$ where $\varepsilon>0$. Then $g$ and $g^{(k)}, k=1,2, \ldots$, have only negative zeros, and if this implies $g \in \mathcal{U}$, then clearly $f \in \mathcal{U}$. Proceeding under this assumption then, we set $m=0$ in (3) and next observe that since $f \in \mathcal{U}_{0}, f(z) \neq c e^{\sigma z}$, either $f$ or $f^{(k)}, k=1,2, \ldots$, must have some (negative) zeros. Also, if

$$
f(z)=1+\sum_{n=1} c_{n} \frac{z^{n}}{n !}
$$

then

$$
c_{n}=f^{(n)}(0) \neq 0, \quad n=1,2, \ldots ;
$$

moreover, it cannot be the case that $c_{n} c_{n+1}<0$ for $n=0,1,2, \ldots$ for then

$$
(-1)^{k} f^{(k)}(-x)=\sum_{n=0}(-1)^{n+k} c_{n+k} \frac{x^{n}}{n !}>0 \text { for } x=\operatorname{Re} z>0
$$

and $f^{(k)}$ cannot have any negative zeros $k=0,1,2, \ldots\left(f^{(0)}=f\right)$. Thus, there is at least one nonnegative integer $n$ for which $c_{n} c_{n+1}>0$; choose such an $n$ and denote it by $k$, so that

$$
c_{k} c_{k+1}>0 \text {. }
$$

Since, as was pointed out above, $f \in \mathcal{Q}_{0}$ implies $f^{(k)} \in \mathcal{Q}_{0}$, we write

$$
f^{(k)}(z)=c_{k} e^{-\gamma_{k} z^{2}+\sigma_{k} z} \Pi_{k}(z)
$$

"where $\gamma_{k} \geqslant 0, \sigma_{k}$ is a real constant, and $\Pi_{k}$ is a canonical product, finite or infinite, of genus $\leqslant 1$ with only negative zeros. Taking the logarithmic 
derivative of (7), we have

$$
\frac{f^{(k+1)}}{f^{(k)}}(z)=-2 \gamma_{k} z+\sigma_{k}+\frac{\Pi_{k}^{\prime}}{\Pi_{k}}(z) .
$$

We now need the following elementary facts about the growth of canonical products of finite genus with only negative zeros: If $P(z)$ is a canonical product of genus $q$ with only negative zeros $\left\{-a_{n}\right\}, a_{n}>0$, then

$$
\frac{P^{\prime}}{P}(z)=(-1)^{q} z^{q} \sum_{n} \frac{1}{a_{n}^{q}\left(z+a_{n}\right)}, \quad z \neq-a_{n},
$$

from which it follows readily that for $x=\operatorname{Re} z$

$$
\left|\frac{P^{\prime}}{P}(x)\right|=o\left(x^{q}\right) \quad(x \rightarrow+\infty)
$$

and

$$
\lim _{x \rightarrow+\infty} \frac{P^{\prime}}{P}(x)=-\infty \quad(q \text { odd }) .
$$

We will now establish (i)-(iii). Since, as is easily verified, $\gamma_{k}=\gamma$ and genus $\Pi_{k}=$ genus $\Pi,{ }^{3}(8)-(10)$ imply that if either $\gamma>0$ or $\Pi$ is of genus 1 then

$$
\lim _{x \rightarrow+\infty} \frac{f^{(k+1)}}{f^{(k)}}(x)=-\infty \quad(x=\operatorname{Re} z) .
$$

Further, (5) and (6) imply that

$$
\frac{f^{(k+1)}}{f^{(k)}}(0)>0 .
$$

It now follows from (11) and (12) that $f^{(k+1)}$ has a positive zero, which is a contradiction. Thus $\gamma=\gamma_{k}=0, \Pi$ and $\Pi_{k}$ are of genus 0 , and, by (3) (with $m=0$ ) and (7)

$$
f(z)=e^{\sigma z} \Pi(z), \quad f^{(k)}(z)=c_{k} e^{\sigma_{k} z} \Pi_{k}(z),
$$

where, as is easily verified, $\sigma=\sigma_{k}$. It then follows from (8) (with $\gamma_{k}=0$ ) and (9) (with $q=0$ ) that if $\sigma=\sigma_{k}<0$, then

$$
\lim _{x \rightarrow+\infty} \frac{f^{(k+1)}}{f^{(k)}}(x)=\sigma_{k}<0 \quad(x=\operatorname{Re} z) .
$$

Reasoning as above, (13) yields a contradiction. Thus $\sigma \geqslant 0$, and the proof of Theorem $\mathrm{B}$ is complete.

REMARK 1. If we alter the hypothesis of Theorem B by dropping the condition that $f$ be real but requiring instead that $f$ be of finite order, we can then use Theorem 2 of [1] and the above proof to conclude that either $f(z)=a e^{b z}, a$ and $b$ constants, or $f \in \mathcal{Q}$.

\footnotetext{
${ }^{3}$ If $f \in \mathscr{Q}_{0}$, then $f^{\prime}$ has only real zeros and $\left(f^{\prime} / f\right)^{\prime}(x)<0$. Thus $f^{\prime}$ has exactly one zero, a simple one, between two consecutive zeros of $f$, so that the genus of $\pi_{1}=$ genus of $\pi$. A simple growth argument shows that $\gamma_{1}=\gamma$. Introduction completes the argument. In the case $\gamma=0$, cf. E. Borel, Lecons sur les fonctions entières, Paris, 1921, p. 32.
} 
Remark 2. Theorem B is false if it is only required that $f$ and $f^{(n)}$, $n=1,2, \ldots, N$, for some $N$ have only real nonpositive zeros. To see this, let $f$ be of the form (3) with only real nonpositive zeros, $f \notin$ Q. By Laguerre's inequality, $\left[f^{(n)}(z) / f^{(n-1)}(z)\right]^{\prime}<0$ for $z \neq$ zero of $f^{(n-1)}$; thus, if we denote by $z_{1 N}$ the largest zero of $f^{(N)}$, it is clear that all zeros of $f$ and $f^{(n)}$ lie in $(-\infty$, $\left.z_{1 N}\right], n=1,2, \ldots, N$. If $z_{1 N}>0$, let $g(z)=f\left(z+z_{1 N}\right)$ and if $z_{1 N}<0$, let $g(z)=f(z)$. In either case $g$ and $g^{(n)}, n=1,2, \ldots, N$, have only real nonpositive zeros; however, $g \notin \mathscr{U}$.

ADDED IN PROOF. Part of our proof involved showing that if $f \in \mathcal{U}_{0}$ and $f^{(k)}$ has only real nonpositive zeros for all $k$, then $\gamma=0$ in (1). This also follows from a theorem of Edrei [Scripta Math. 22 (1956), Theorem 1] which readily implies the following

THEOREM C. Let $F(z)=e^{-\gamma z^{2}} G(z), \gamma>0$ and $G \in \mathscr{U}_{0}$ of genus $<1$. Then the zeros of the successive derivatives of $f$ are everywhere dense on the real axis.

\section{REFERENCES}

1. S. Hellerstein and J. Williamson, Derivatives of entire functions and a question of Pólya, Trans. Amer. Math. Soc. 227 (1977), 227-249.

2. __ Derivatives of entire functions and a question of Pólya. II, Trans. Amer. Math. Soc. (to appear).

3. E. Laguerre, Sur les fonctions du genre zéro et du genre un, C. R. Acad. Sci. 98 (1882); Oeuvres 1 (1898), $174-177$.

4. G. Pólya, Uber Annäherung durch Polynome mit reellen Wurzeln, Rend. Circ. Mat. Palermo 36 (1913), 279-295.

5. $\longrightarrow$ Sur une question concernant les fonctions entières, C. R. Acad. Sci. Paris 158 (1914), $330-333$.

6. __ Bemerkung zur Theorie der ganzen Funktionen, Jber. Deutsch. Math. Verein. 24 (1915), 392-400.

Department of Mathematics, University of Wisconsin, Madison, Wisconsin 53706

Department of Mathematics, University of Hawail, Honolulu, Hawail 96822 